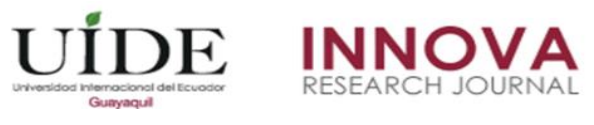

INNOVA Research Journal, ISSN 2477-9024

(Marzo, 2017). Vol. 2, No.3 pp. 58-68.

DOI: https://doi.org/10.33890/innova.v2.n3.2017.135

URL: http://revistas.uide.edu.ec/index.php/innova/index

Correo: innova@uide.edu.ec

\title{
Seguridad y salud ocupacional en Ecuador: Contribución normativa a la responsabilidad social organizacional
}

\author{
Occupational safety and health in Ecuador: \\ Normative contribution to organizational social responsibility
}

\author{
María Pilar Martínez Barranco \\ Universidad Complutense de Madrid, España \\ Edison Daniel Yandún Burbano \\ Universidad Internacional SEK Ecuador
}

Autor para correspondencia: mariapilarmartinez@ucm.es, daniel.yandun@uisek.edu.ec.

Fecha de recepción: 3 de enero de 2017 - Fecha de aceptación: 20 de Febrero de 2017

Resumen: La Responsabilidad Social Organizacional es el nuevo paradigma de gestión estratégica de las corporaciones. Dentro de sus grupos de interés fundamentales se encuentran los trabajadores y consecuentemente, su seguridad y salud. La Responsabilidad Social es de carácter voluntario. Objetivo: conocer el alcance de la legislación ecuatoriana en materia de Seguridad y Salud Ocupacional (SSO) analizando y comprobando si los contenidos exigibles en la ISO 26000 (6.4.6.2) están reflejados en la normativa de Ecuador, para así poder diferenciar en futuros estudios, las acciones que sobrepasan la norma e identificarlas como "buenas prácticas" en sostenibilidad. Material-Métodos: Estudio comparativo de tipo observacional a partir de fuentes de información secundarias; normativa legal ecuatoriana vigente aplicable sobre Seguridad y Salud Ocupacional y, la Norma ISO 26000 (Guía de Responsabilidad Social) en su apartado 6.4.6.2. Se ha elegido esta norma por estar reconocida a nivel internacional y, Ecuador fue parte integrante en su elaboración. Resultados: Cuadro comparativo referido en cada punto de la Guía ISO 26000 con su respectiva legislación ecuatoriana aplicable. Conclusiones: Se puede determinar que la normativa legal ecuatoriana observa todas y cada una de las recomendaciones indicadas en la Guía. Palabras Clave: Responsabilidad Social Organizativa; Seguridad y Salud Ocupacional; ISO 26000; Legislación; Ecuador

Abstract: Corporate Social Responsibility is the new paradigm of corporate strategic management. Within its fundamental interest groups are workers and consequently their health and safety. Granted that Social Responsibility is voluntary in nature. Objective: to know the scope of the Ecuadorian legislation on Occupational Health and Safety by analyzing and verifying whether the contents required by ISO 26000 (6.4.6.2) are reflected in the regulations of Ecuador, in order to differentiate in future studies, the actions surpass the norm and identify them as "good practices" in sustainability. Material-Methods: comparative observational study using secondary information sources; Current legal regulations applicable to Occupational Safety and Health, and ISO 26000 Social Responsibility Guide (section 6.4.6.2). This standard has been chosen because it is internationally recognized and Ecuador was an integral part of its development. Results: Comparative table referring to each point of the ISO 26000 Guide and the respective Ecuadorian legislation applicable to it. Conclusions: It can be determined that Ecuadorian legal regulations observe each and every one of the recommendations indicated in the Guide. 
Key Words: Corporate Social Responsibility; Occupational Health and Safety; ISO 26000; Legislation; Ecuador

\section{Introducción}

El siglo XXI se caracteriza por la Globalización, en este contexto la Responsabilidad Social Empresarial (RS) se afirma como una respuesta ante la ciudadanía por los múltiples impactos que generan las organizaciones en los ámbitos social, económico y medioambiental (Comisión Europea, 2011). Con el fin de aproximar el concepto de Responsabilidad Social y sus diversas nomenclaturas (Responsabilidad Social Empresarial, Responsabilidad Social Corporativa, Responsabilidad Social Organizacional,...) se comienza este estudio mediante la exposición de las definiciones más difundidas para, posteriormente, hacer un breve resumen desde su origen en los años 50’ del siglo pasado, con las teorías más relevantes y contenidos, para desembocar al momento actual, en el que su implantación es de carácter básico y fundamental en las organizaciones debido en parte al contexto socioeconómico actual, y al desarrollo de las Tecnologías de la Información y Comunicación (TICs), con la consiguiente disposición al momento de la información y la globalización de la economía.

A día de hoy no existe una definición única consensuada a nivel internacional de la Responsabilidad Social; existen tantas definiciones como organismos involucrados en su estudio, expansión e implementación. Para contextualizar este trabajo se han contemplado las siguientes definiciones:

- "La integración voluntaria por parte de las empresas de las preocupaciones sociales y medioambientales en sus operaciones comerciales y sus relaciones con los interlocutores" (Comisión Europea, 2001)

- $\quad$ "La responsabilidad social de la empresa (RSE) es el reflejo de la manera en que las empresas toman en consideración las repercusiones que tienen sus actividades sobre la sociedad, y en la que afirman los principios y valores por los que se rigen, tanto en sus propios métodos y procesos internos como en su relación con los demás actores. La RSE es una iniciativa de carácter voluntario y que sólo depende de la empresa, y se refiere a actividades que se considera rebasan el mero cumplimiento de la legislación." (Organización Internacional del Trabajo, 2006)

- "La responsabilidad de una organización ante los impactos que sus decisiones y actividades ocasionan en la sociedad y el medio ambiente, mediante un comportamiento ético y transparente que: 1) contribuya al desarrollo sostenible incluyendo la salud y el bienestar de la sociedad; 2) tome en consideración las expectativas de sus partes interesadas; 3 ) cumpla con la legislación aplicable y sea coherente con la normativa internacional de comportamiento; y 4) esté integrada en toda la organización y se lleve a la práctica en sus relaciones" (ISO 26000, 2010).

\section{Origen y Evolución de la Responsabilidad Social}

El origen de la Responsabilidad social proviene de Estados Unidos, a mediados del siglo pasado, Howard R. Bowen, considerado el padre de la Responsabilidad Social, en su libro "Social Responsabilities for de Businessman" la define como "la obligación que tienen los directivos empresariales de establecer políticas, tomar decisiones o seguir líneas de acción que 
sean deseables en términos de los objetivos y valores de nuestra sociedad", incluye una serie de propuestas para que la gestión empresarial involucre los intereses sociales. En esta obra recopiló las diferentes posturas y teorías de los empresarios y economistas de la época. Se pasó del concepto de Responsabilidad Social como filantropía, a una nueva forma de contemplar a las organizaciones como promotoras y cuidadoras de la sociedad donde operan.

Desde ese momento e inicialmente de manera poco representativa, en la siguiente década comienzan los debates acerca de las obligaciones de la empresa con su entorno tanto exterior como interior, aparece el término "stakeholder" o grupos de interés. Joseph W. McGuire en su libro "Business and Society", considera la RS como una actividad más en la gestión empresarial tan importante como las económicas. Keith Davis en 1960, introduce el concepto de retorno de la inversión en RS a largo plazo en su artículo "Can Business Afford to Ignore Social Responsibilities?" en el que afirma que el poder del empresario se puede perder si no lleva a la práctica acciones de responsabilidad social; posteriormente en un nuevo artículo (1967) "Understanding the social responsability puzzle: what does the businessman owe to society?" plantea la dependencia real entre empresa y sociedad, y, la importancia en la supervivencia de la empresa si la sociedad no le otorga ese poder.

Es en los años 70' del siglo XX cuando se aborda la RS en los círculos empresariales. Milton Friedman publica un artículo en el New York Times en el que asevera que "La Responsabilidad Social de las empresas es incrementar los beneficios", de esta forma, la sociedad verá mejorada su calidad de vida ya que la empresa contribuirá a su crecimiento mediante el aporte de impuestos y creación de empleo, fue el propulsor de la "Shareholder Theory". Fue una década con gran profusión de teorías y declaraciones que terminó con la propuesta de Archie B. Carroll publicada en su artículo " A three-dimensional conceptual model of corporate performance" en 1979, aún vigente, de un modelo de RS de gestión empresarial basado en cuatro elementos: económico, legal, ético y discrecional (filantropía).

Durante los años 80' y 90' la Responsabilidad Social comienza a ser un asunto principal en los negocios, los escándalos y riesgos en los que las organizaciones están involucradas son de conocimiento público debido a la aparición de las TIC y la globalización de los negocios. En 1984, R. Edward Freeman publica "Strategic Management: A Stakeholder Approach", recopilación de varios autores y exposición de la denominada "Stakeholder Theory" en la que se centra la atención en los grupos de interés, incluyendo a todos aquellos que se ven afectados por la actividad de la empresa, no sólo los accionistas como defiende Friedman.

Desde finales del siglo XX y la primera década del XXI son numerosas las publicaciones y estudios que se hacen eco de la importancia en términos de sostenibilidad de gestionar las empresas basado en un sistema integral actuando en torno a las tres dimensiones que comprende la responsabilidad Social (Naciones Unidas, 2013):

1. D. Económica: viabilidad y capacidad para contribuir al desarrollo económico, tanto particular como de la sociedad donde opera.

2. D. Social: los impactos de la actividad de la empresa hacia los trabajadores (condiciones de trabajo, SSO, etc.), los proveedores, los clientes, las comunidades locales y la sociedad en general, y defensa de Derechos Humanos.

3. D. Medioambiental: busca la sostenibilidad mediante la compatibilidad entre la actividad social de la empresa y la preservación de la biodiversidad y de los ecosistemas. 
En este estudio se abordará una parte de la dimensión social, la referente a la SSO. Los trabajadores son definidos como stakeholders "primarios" (Clarckson, 1995), o "internos" (Navarro García, 2012), ya que mantienen una relación contractual con la organización, influyen en los resultados económicos y sin ellos la empresa no existiría.

\section{Responsabilidad Social en Ecuador}

La Responsabilidad social en Ecuador desde finales del siglo XIX ha sido entendida mayoritariamente como actos filantrópicos que realizaban los propietarios de las empresas a favor de sus trabajadores y familias. En el siglo XX las empresas multinacionales que se afincaron en Ecuador trajeron sus incipientes políticas de RS, tal y como las venían gestionando en sus matrices.

La Constitución de la República del Ecuador recoge el "Bien Común” en un número considerable de artículos y en concreto con su política del "buen vivir", la construcción de un estado sostenible. En lo que llevamos del siglo XXI se han creado organismos específicos para el desarrollo de la RS tanto de carácter privado en forma de asociaciones y fundaciones (CERES, EKOS, IRSE), como públicos, liderando la Municipalidad de Quito mediante sus Ordenanzas (n⿳333/2010 y nº 084/2015), creándose el Consejo Metropolitano de Responsabilidad Social (CMRS), conformado por 17 organizaciones público-privadas cuyo objetivo es motivar y concienciar a la población, concretamente, la 084 va dirigida desde el ciudadano hacia las instituciones, reflejando el poder de la ciudadanía para la implantación de la RS, a diferencia de otras políticas que pretender llegar al ciudadano desde las empresas privadas y las administraciones públicas.

Reflejo del interés sobre la sostenibilidad fue la participación en la elaboración de la ISO 26000 (2010), el establecimiento de la Red Nacional del Pacto Global (2011) y, las celebraciones del "Primer Foro de Quito de Responsabilidad Social" (2011) y la "Primera Cumbre Mundial de Responsabilidad Social (2013). Así mismo, en el año 2012, se han realizado diversos estudios tanto sobre el conocimiento y percepción de la RS de las empresas y ciudadanos en Ecuador; los más importantes cuantitativa y cualitativamente son el realizado por el Comité de Responsabilidad Corporativa de la Cámara de Comercio Ecuatoriano-Americana, en alianza con 13 organizaciones del sector público, sector privado y la academia, y, el Estudio de Responsabilidad Social de Empresas del Ecuador elaborado por la Fundación AVINACooperación Alemana al Desarrollo GIZ-IDE Business School.

\section{Seguridad y Salud Ocupacional}

La Organización Mundial de la Salud (OMS) define la Salud Ocupacional como "una actividad multidisciplinaria dirigida a promover y proteger la salud de los trabajadores mediante la prevención y el control de enfermedades y accidentes y la eliminación de los factores y condiciones que ponen en peligro la salud y la seguridad en el trabajo" (Organización Mundial de la Salud, 1995). Un avance importante en el que se incluye la prevención, control y eliminación de factores con respecto a la emitida en 1948: "un estado de bienestar total que incluye el bienestar físico, mental y social, y no la mera ausencia de enfermedad o trastornos". 
La definición global adoptada por el Comité Mixto OIT/OMS de Salud en el Trabajo en su primera reunión (1950) y revisada en su duodécima reunión (1995), "la finalidad de la salud en el trabajo consiste en lograr la promoción y mantenimiento del más alto grado de bienestar físico, mental y social de los trabajadores en todos los trabajos". Según la Asociación Internacional de la Higiene Ocupacional (IOHA), la higiene ocupacional es la ciencia de la anticipación, el reconocimiento, la evaluación y el control de los riesgos derivados del lugar de trabajo, o producidos en el mismo, que podrían perjudicar la salud y el bienestar de los trabajadores, y además tiene en cuenta la posible repercusión en las comunidades cercanas y el medio ambiente en general. La Sociedad Americana de Ingenieros de Seguridad define este concepto en términos similares a los de la IOHA, aunque hace hincapié en la «eliminación progresiva» de los riesgos y en los programas de prevención de los mismos. (Organización Internacional del Trabajo, 2003).

La SSO de los empleados influye en la de sus familiares, colaboradores y sociedad en general, su introducción en la estrategia empresarial reporta eficiencia, optimiza recursos y genera beneficios económicos derivados tanto del ahorro de costes como de la reducción de contingencias y sanciones, incrementa la imagen reputacional, y la fidelización del personal mediante la generación de sentimiento corporativo (Greening \& Turban, 2000) así como crea ventaja competitiva derivada de otros atributos intangibles.

Los empleados son el grupo de interés de mayor impacto en la organización, su satisfacción se refleja en la productividad y depende tanto de las condiciones físicas de su tarea como de las psicológicas. Con respecto a las primeras, éstas se refieren tanto al lugar de trabajo, edificios e instalaciones (iluminación, temperatura, ergonomía, etc.) como a los materiales empleados, maquinaria, utillaje, etc. Existe numerosa regulación para la prevención de riesgos físicos que emana de los poderes públicos, así como mecanismos y medios de protección para minimizar, prevenir y atenuar las consecuencias adversas derivadas de la manipulación o estancia en el puesto de trabajo. Así mismo, las condiciones psicológicas deben ser contempladas, la Psicología de la Salud Ocupacional (PSO) es la disciplina que comprende la aplicación de la psicología a la mejora de la calidad de vida laboral, y de proteger y promover la seguridad, la salud y el bienestar de los trabajadores (National Institute of Occupational Safety and Health, NIOSH) (Salanova, Martínez, Cifre, \& Llorens, 2009).

\section{Responsabilidad Social en Seguridad y Salud Ocupacional}

La SSO es un asunto de gran interés dentro de la Responsabilidad Social, se articula dentro de la Dimensión Social y está vinculada necesariamente con las condiciones de trabajo de los empleados. Las empresas se deben comprometer a lograr elevados estándares de salud y bienestar en sus empleados, prestando especial atención al clima organizacional y la salud ocupacional, entre otros aspectos (Alas \& Tafel, 2008).

No se debe plantear que la salud de los empleados es un medio para alcanzar una buena imagen reputacional, la finalidad es lograr un ambiente de trabajo saludable y que éste se convierta en un valor estratégico de la compañía. Las consecuencias de una mala política 
suponen un coste social y económico importante, debido a un menor desempeño, absentismo, rotación, etc., debido fundamentalmente a la insatisfacción laboral.

Desde los años 90' del siglo pasado, las condiciones de trabajo han variado sustancialmente: innovación tecnológica, flexibilidad en el empleo, incorporación de la mujer, diversidad, internacionalización de las empresas, fusiones, subcontratación, etc. Surgen nuevos factores que incrementan los riesgos para la salud de los trabajadores, entre ellos, los desplazamientos al lugar de trabajo y de especial relevancia los nuevos factores de riesgo psicosocial, que provienen del ritmo, logro de metas y el aprendizaje continuo en un estado de competencia constante como uno de los principales estresores.

La expansión de las TICs ha dado a conocer las condiciones de seguridad y salud en los centros de trabajo en países en vía de desarrollo. Escándalos como el incendio de una fábrica textil en Bangladesh (2013), y muchos otros, han puesto en evidencia las condiciones de trabajo en entornos no saludables e inseguros.

Para facilitar la integración de la Responsabilidad Social en las organizaciones existen múltiples instrumentos, unos como guía de actuación (ISO 26000, GRI), normas internacionales (OSHAS 18000, AccountabilityAA1000), pactos internacionales (Global Compact), Memorias de Sostenibilidad, Directrices de la OCDE, informes certificables (SGE-21), Códigos Éticos/Conducta/Buen Gobierno, Indicadores (Ethos, sectoriales, locales...). La elección de la Norma-Guía ISO 26000 como marco de referencia en este trabajo se debe fundamentalmente a que es de aplicación en todo tipo de empresas independientemente de su tamaño, régimen jurídico o sector en el que opera; recoge las mejores prácticas en concordancia con la OIT, el Global Compact y la OCDE, y facilita la implementación de la RS a través de sus recomendaciones. Ecuador mediante el Instituto Ecuatoriano de Normalización (INEN) fue uno de los 83 países participantes activos en la elaboración de la misma.

El objetivo del presente estudio fue identificar y comparar la normativa legal ecuatoriana sobre SSO con la Norma internacional- Guía ISO 26000 (Guía de Responsabilidad Social) en su apartado 6.4.6.2. Para verificar en qué medida la integración de la Responsabilidad Social es obligatoria en las empresas del país.

\section{Material - Métodos}

Estudio comparativo de tipo observacional a partir de fuentes de información secundaria; normativa legal ecuatoriana vigente aplicable sobre SSO y la Norma internacional ISO 26000 (Guía de Responsabilidad Social) concretado en su apartado 6.4.6.2.

Se procedió a la identificación y análisis de la normativa legal (noviembre 2016) en esta materia, para su posterior comparación en cada una de las recomendaciones incluidas en el apartado indicado de la Norma ISO 26000. 


\section{Tabla 1. Legislación aplicable de Ecuador}

\begin{tabular}{ll}
\hline Legislación: nomenclaturas \\
\hline C.R.E. & Constitución de la República del Ecuador \\
\hline D-584 & $\begin{array}{l}\text { Decisión 584. } \\
\text { Instrumento Andino de Seguridad y Salud en el Trabajo }\end{array}$ \\
\hline R-957 & $\begin{array}{l}\text { Resolución 957. } \\
\text { Reglamento del Instrumento Andino de Seguridad y Salud en el Trabajo }\end{array}$ \\
\hline C.T. & Código del Trabajo \\
\hline D.E.-2393 & $\begin{array}{l}\text { Decreto Ejecutivo 2393. } \\
\text { Reglamento de Seguridad y Salud de los Trabajadores y Mejoramiento del Medio Ambiente } \\
\text { de Trabajo }\end{array}$ \\
\hline C.D. 513 & $\begin{array}{l}\text { Resolución N } \text { C.D. 513. } \\
\text { Reglamento Seguro General de Riesgos del Trabajo }\end{array}$ \\
\hline A-1404 & Reglamento para el Funcionamiento de los Servicios Médicos de Empresas \\
\hline A.M.- 398 & Acuerdo Ministerial 398 VIH-Sida - Ministerio Del Trabajo \\
\hline
\end{tabular}

\section{Resultados}

Los resultados obtenidos en base a las recomendaciones de la ISO 26000 en su cláusula 6.4.6.: "Asunto sobre prácticas laborales: salud y seguridad ocupacional” y la legislación vigente ecuatoriana se detallan en la siguiente tabla:

Tabla 2. Comparativa ISO 26000 - 6.4.6.2 vs Referencial Legal de Ecuador

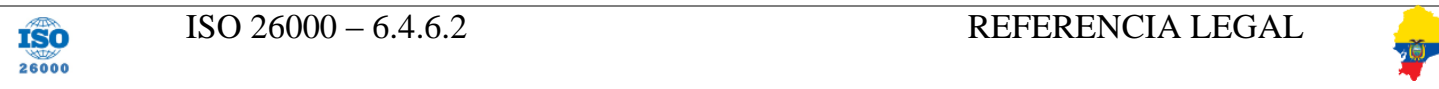

1. Comprender y aplicar principios de

- $\quad$ D-584. CAPÍTULO III. Artículo 11. gestión de la salud y la seguridad,

- C.D.-513. CAPÍTULO XI, Artículo 53. incluyendo la jerarquía de controles: eliminación, sustitución, controles de ingeniería, controles administrativos, procedimientos laborales y equipos protección personal.

2. Desarrollar, implementar y mantener una política de salud y seguridad ocupacional basada en el principio de que normas sólidas en materia de salud y seguridad y el desempeño de la organización se apoyan y refuerzan mutuamente.

3. Analizar y controlar los riesgos para la salud y la seguridad derivados de sus actividades.

- C.R.E. CONSTITUCIÓN DEL REPÚBLICA DEL ECUADOR, Articulo 326. Numeral 5.

- D-584. CAPÍTULO III. Artículo 11 Literal a.

- R-957. CAPÍTULO I, Artículo 1, Literal a.1.

- D-584. CAPÍTULO III. Artículo 11. Literal b) y c)

- D.E.-2393. Art. 15. Numeral 2 literal a) y b).

- R-957. Artículo 1, Literal b).

- C.D.-513. CAPÍTULO XI, Artículo 53 literal a),b), c), d). Artículo 55.

4. Comunicar la exigencia que establece que - D-584. Art. 24. Artículo 11, literal e) los trabajadores deberían seguir todas las
- D.E.-2393. Artículo 13. 
prácticas de seguridad en todo momento y asegurarse de que los trabajadores siguen los procedimientos adecuados.

5. Proporcionar el equipo de seguridad

- D-584. Artículo 11, literal c)

- D.E.-2393. Título VI: Protección Personal. Artículos 176, 177, 178, 179, 180, 181, 182, 183. protección personal, para la prevención de lesiones, enfermedades y accidentes laborales, así como para el tratamiento de emergencias

6. Registrar e investigar todos los incidentes y problemas en materia de seguridad y salud, con el objeto de minimizarlos o eliminarlos.

7. Abordar las maneras específicas en que los riesgos de salud y seguridad ocupacional (OSH, por sus siglas en inglés) afectan de forma diferente a mujeres (como, por ejemplo, a las embarazadas, las que han dado a luz recientemente o las que se encuentran en periodo de lactancia) y a hombres, o a trabajadores en circunstancias concretas, como, por ejemplo, las personas con discapacidad, los trabajadores sin experiencia o los jóvenes.

8. Proporcionar protección equitativa en salud y seguridad a trabajadores de tiempo parcial y temporales, así como a los trabajadores subcontratados;

9. Esforzarse por eliminar los riesgos psicosociales en el lugar de trabajo que contribuyen o provocan estrés y enfermedades.

10. Proporcionar la formación adecuada en todos los aspectos pertinentes a todo el personal.

11. Respetar el principio de que las medidas de salud y seguridad en el lugar de trabajo no deberían involucrar gastos monetarios para los trabajadores.

12. Basar sus sistemas de salud seguridad y medio ambiente en la participación de los trabajadores afectados y reconocer y respetar los derechos de los trabajadores a:

- obtener información completa y precisa, en el momento oportuno, concerniente a los riesgos de salud y seguridad y de las mejores prácticas de empleadas para hacer frente a esos riesgos;

- Consultar y ser consultado, libremente, acerca de todos los aspectos de salud y seguridadrelacionados con su trabajo;

- Rechazar un trabajo sobre el que razonablemente pueda pensarse que representa un peligro inminente o serio
- C.R.E. CONSTITUCIÓN DE LA REPÚBLICA DEL ECUADOR, Artículo 326. Numeral 5.

- D-584. Artículos 18, 19, 20, 21.

- D.E.-2393. Artículos 11 y 12.

- C.R.E. CONSTITUCIÓN DE LA REPÚBLICA DEL ECUADOR, Artículo 331.

- A-1404. CAPÍTULO IV Art. 11, Numeral, Literal c)

- $\quad$ D-584. Art. 11 literal h), I) Art. 18, 19, 20 y 23.

- A.M.- 398 VIH-SIDA

- D.E.-2393. Art. 11, numeral 5).
Comités Paritarios de Salud y Seguridad

- R-957. Artículos 13 y 14.

- D.E.-2393. Artículo 14.

Unidad De Seguridad y Salud

- D.E.-2393. Artículo 15, Numeral 1.

- D-584. CAPÍTULO III, Artículo 11, Literal a). Servicio Médico Permanente

- C.T. Artículo 430, Numeral 2.

- D.E.-2393. Artículo 16.

- A-1404.

Reglamento Interno de Seguridad y Salud eneEl Trabajo

- C.T. Artículo 434.

Plan Mínimo De Seguridad Y Salud

- $\quad$ D-584. CAPÍTULO III, Artículo 11. MAPA DE RIESGOS 
para su vida o salud o para la vida y la salud de otros;

- Buscar asesoramiento externo de organizaciones de trabajadores y empleadores así como de otras que tengan conocimiento del tema;

- Informar a las autoridades competentes sobre asuntos de salud y seguridad;

- Participar en decisiones y actividades relacionadas con la salud y la seguridad, incluyendo la investigación de incidentes $\mathrm{y}$ accidentes, $\mathrm{y}$

- Estar libre de las amenazas de represalias por llevar a cabo cualquiera de las acciones mencionadas

\section{Fuente: Elaboración de los autores}

\section{Conclusiones-Discusión}

El espíritu de la norma ISO 26000 en el Asunto 4 sobre prácticas laborales es promover y mantener el más alto grado de bienestar físico, mental y social de los trabajadores y prevenir los daños en la salud provocados por las condiciones laborales; ante esto, Tras la realización de la comparación respectiva se concluye que la legislación ecuatoriana mediante los cuerpos legales existentes cumple con lo manifestado por ISO 26000 que es de carácter voluntario. Es decir que todas las organizaciones ecuatorianas que cumplen con la parte legal en S.S.O. cumplen con los principios enmarcados en la norma ISO 26000.

Así en Ecuador es de cumplimiento obligatorio el gestionar la seguridad y salud laboral por parte de la alta dirección en donde es clave su compromiso; entre otras cuestiones, el formular la Política de Seguridad y Salud pues se marca un objetivo empresarial en esta materia garantizando recursos y, manifestando el compromiso de cumplir con la legislación técnico legal vigente en el país y el de garantizar las óptimas condiciones de trabajo.

La dirección empresarial es responsable de la creación y supervisión de la Unidad de Seguridad y Salud, esto aplica para empresas con 100 o más trabajadores o centros de trabajo calificados de alto riesgo cuando cuenten con más de 50 trabajadores. Esta Unidad de Seguridad y Salud que se forma desde un punto de vista preventivo, es dirigida por un profesional técnico en la materia, el cual se encarga de identificar, medir, evaluar y controlar los riesgos laborales sean estos en la fuente, medio y receptor y, crear programas que incluyan políticas preventivas. En la parte de Salud Ocupacional existe un servicio médico de empresa el cual se enfoca en prevenir posibles enfermedades laborales.

El instrumento legal de gestión lo desarrolla la Unidad de Seguridad y Salud con la aprobación de la Dirección y el Ministerio del Trabajo: es el Reglamento de Seguridad y Salud, en el cual prima el identificar y evaluar los riesgos existentes en la organización y plantea el control de los mismos, crea responsabilidades preventivas a todo nivel así como los derechos y obligaciones de los miembros de una organización. 
La participación de los trabajadores es fundamental en materia de seguridad y salud para lo cual las organizaciones que cuenten con más de quince trabajadores deben organizar un organismo paritario denominado Comité y/o Sub Comités de Seguridad y Salud conformado por representantes de los trabajadores y del empleador el cual se encarga entre otras funciones, de promover la observancia de las disposiciones sobre prevención de riesgos profesionales. Al finalizar un periodo se presenta a la Dirección y a los organismos de control como el Instituto Ecuatoriano de Seguridad Social o Ministerio de Trabajo los indicadores de gestión y actividades realizadas por los organismos paritarios.

Como continuación a la investigación, sería conveniente averiguar si estas acciones se están cumpliendo por parte de las organizaciones, así como si las Administraciones Públicas ecuatorianas implicadas realizan su debida vigilancia. En la misma línea, el estudio continuará con la detección de acciones/buenas prácticas activas de carácter voluntario realizadas por las empresas ecuatorianas no contempladas en la ISO 26000 (6.4.6.2), con el fin de hacerlas públicas para su conocimiento y puesta en marcha en otras organizaciones.

La Agencia Europea para la Seguridad y Salud en el trabajo propone como vía de mejora la PSLT (Promoción de la salud en el lugar de trabajo para los trabajadores), que comprende 4 fórmulas de avance en la calidad de vida del trabajador: 1: La mejora del método de organización del trabajo; 2: La mejora del entorno de trabajo; 3: Fomentar la participación de los empleados en actividades saludables; 4: Fomentar el desarrollo personal. Todas ellas implican el ir más allá del mero cumplimiento de los requisitos legales en materia de seguridad y salud en el trabajo correspondiéndose con una de las características fundamentales de la RS, su carácter voluntario y de evitación de impactos, de esta forma, las empresas contribuyen de manera activa la mejora de la salud y el bienestar general de sus trabajadores (Agencia Europea para la Seguridad y la Salud en el Trabajo, 2010).

\section{Agradecimiento}

Programa Universidad Complutense de Madrid - Santander Universidades

\section{Bibliografía}

Agencia europea para la Seguridad y la Salud en el Trabajo. (2010). Promoción de la salud en el trabajo para los trabajadores. Recuperado el 2 de 12 de 2016, de http://osha.europa.eu/es/publications/factsheets/94

Alas, R., \& Tafel, K. (2008). Conceptualizing the dynamics of social responsibility. Evidence from a case study of Estonia. Journal of Business Ethics (81 (2)), 371-385.

Clarckson, M. (1995). A stakeholder framework for analyzing and evaluating corporate social performance. Academy of Management Review (20(1)), 92-117.

Comisión Europea. (2001). Libro Verde, Fomentar un marco europeo para la responsabilidad social de las empresas. Recuperado el 15 de noviembre de 2016, de http://eurlex.europa.eu/legal-content/ES/TXT/?uri=CELEX:52001DC0366 
Comisión Europea. (2011). Estrategia renovada de la UE 2011-2014 sobre la responsabilidad social de las empresas. Bruselas.

Greening, D., \& Turban, D. (2000). Corporate social performance as a competitive advantage in attracting a quality workfoce. Business and Society (39(3)), 254-281.

ISO 26000. (2010). Recuperado el 25 de 11 de 2016, de https://www.iso.org/obp/ui\#iso:std:iso:26000:ed-1:v1:es

Naciones Unidas. (2013). Objetivos de Desarrollo del Milenio. Recuperado el 26 de noviembre de 2016, de http://www.un.org/es/millenniumgoals/pdf/mdg-report-2013-spanish.pdf

Navarro García, F. (2012). Responsabilidad Social Corporativa. Teoría y práctica. Madrid: ESIC Editorial.

Organización Internacional del Trabajo. (2003). Actividades normativas de la OIT en el ámbito de la seguridad y la salud en el trabajo: estudio detallado para la discusión con miras a la elaboración de un plan de acción sobre dichas actividades. 91 . Conferencia Internacional del Trabajo. Suiza: OIT. Recuperado el 18 de 11 de 2016, de http://www.ilo.org/public/spanish/standards/relm/ilc/ilc91/pdf/rep-vi.pdf

Organización Internacional del Trabajo. (2006). Iniciativa In Focus sobre responsabilidad social de la empresa. Recuperado el 25 de 11 de 2016, de http://www.ilo.org/public/libdoc/ilo/GB/295/GB.295_MNE_2_1_span.pdf

Organización Mundial de la Salud. (1995). Salud Ocupacional para Todos. Recuperado el 15 de 11 de 2016, de http://apps.who.int/iris/bitstream/10665/42109/1/951802071X_spa.pdf

Salanova, M., Martínez, I., Cifre, E., \& Llorens, S. (2009). La salud ocupacional desde la perspectiva psicosocial: aspectos teóricos y conceptuales. Psicología de la Salud Ocupacional, 27-62. 